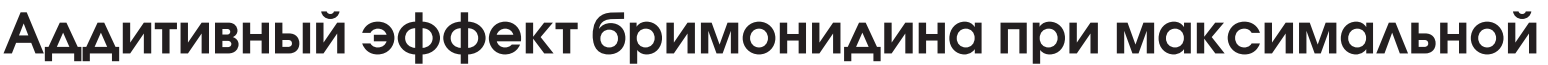 меАикаментозной терапии у пациентов с первичной открытоугольной глаукомой
}

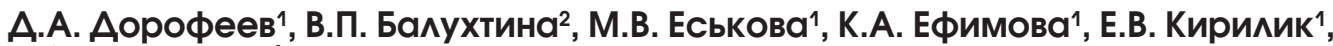 \\ К.О. Аукьянова ${ }^{1}$ \\ "МАУЗ «ГКБ № 2», Челябинск, Россия \\ 2ФГБОУ ВО ЮУГМУ Минздрава России, Челябинск, Россия
}

\begin{abstract}
РЕЗЮМЕ
Цель исследования: оценка аддитивного эффекта снижения внутриглазного давления (ВГД) бримонидином 0,2\% для достижения давления цеели при усилении максимального режима гипотензивной терапии первичной открытоугольной глаукомы (ПОУГ).

Материал и методы: в исследовании приняли участие 63 пациента (63 глаза) с развитой стадией ПОУГ и субкомпенсацией ВГД на фоне терапии аналогами простагландинов вместе с фиксированной комбинацией ингибитора карбоангидразы и бета-блокатора. Всем пациентам дополнительно был назначен бримонидин 0,2\%. Через 1 мес. в зависимости от достигнутого уровня ВГД пац̨иенты были разделены на 2 группы: 1-я группа - пацциенты с достигнутым давлением иели, они остались под наблюдением; 2-я группа - пациенты с субкомпенсированным ВГД, им была проведена синустрабекулэктомия. Оиенка ВГД проходила с использованием тонометра іСаге и эластотонометрии.

Результаты исследования: через 1 мес. после назначения а2-адреномиметика в 1-й группе уровень истинного ВГД снизился до 14,0 (9,5; 17,0) мм рт. ст., а во 2-й группе - до 17,0 (13,0; 20,0) мм рт. cm. $(p<0,05)$. На заключительном этапе уровень истинного ВГД в обеих группах сохранялся в пределах цеелевых значений - 13,0 $(11,0 ; 18,5)$ и 13,5 $(9,7 ; 17,2)$ мм рт. ст. соответственно. Динамика ВГД, оцененная различными способами, имела существенные различия: по данным эластотонометрии в 1-й группе снижение составило 5,4\% (-7,1\%; 17,6\%), а во 2-й - 20,7\% (4,4\%; 30,7\%) (p<0,05), по данным тонометра іСаге снижение составило 8,3\% (-11,8\%; 28,6\%) в 1-й группе и $33,3 \%(13,9 \% ; 50,7 \%)$ во 2-й группе.

Заключение: проведенное исследование демонстрирует аддитивный эффект бримонидина 0,2\% при усилении максимального режима гипотензивной терапии развитой стадии ПОУГ. При его назначении необходимо учитывать предполагаемый гипотензивный эффект - дополнительное снижение истинного ВГД на 8\%, и, если это снижение не позволяет достичь цеелевого уровня, планировать оперативное вмешательство. Предпочтительнее проводить измерение тонометром іСаге как более чувствительным к небольшим перепадам офтальмотонуса.

Ключевые слова: бримонидин 0,2\%, максимальная гипотензивная терапия, тонометрия, эластотонометрия, глаукома, синустрабекулэктомия, аддитивный эффект, иелевое давление, субкомпенсаиия.

Для цитирования: Дорофеев Д.А., Балухтина В.П., Еськова М.В. и др. Аддитивный эффект бримонидина при максимальной медикаментозной терапии у пациентов с первичной открытоугольной глаукомой. Клиническая офтальмология. 2021;21(3):129-134. DOI: $10.32364 / 2311-7729-2021-21-3-129-134$
\end{abstract}

\section{Additive effect of brimonidine in maximum tolerated medical therapy for primary open-angle glaucoma}

\author{
D.A. Dorofeev', V.P. Balukhtina'2, M.V. Es'kova', K.A. Efimova', E.V. Kirilik', K.O. Luk'yanova' \\ 'City Clinical Hospital No. 2, Polyclinic № 1, Chelyabinsk, Russian Federation \\ ${ }^{2}$ South Ural State Medical University, Chelyabinsk, Russian Federation
}

\begin{abstract}
Aim: to evaluate additive IOP-lowering effect of brimonidine $0.2 \%$ to achieve target IOP via enhancing maximum tolerated medical therapy for primary open-angle glaucoma (POAG).

Patients and Methods: 63 patients (63 eyes) with advanced POAG and poorly controlled IOP who received prostaglandin analogs and a fixeddose carbonic anhydrase inhibitor/beta-blocker combination were enrolled. All patients were additionally prescribed with brimonidine $0.2 \%$. After a month, patients were divided into two groups based on achieved IOP level. In group 1, target IOP was achieved, and these patients were followed up. In group 2, target IOP was not achieved, and these patients underwent trabeculectomy. IOP was measured by elastotonometry and using the iCare tonometer.

Results: a month after prescribing a2 agonist, true IOP level reduced to 14.0 (9.5; 17.0) $\mathrm{mm} \mathrm{Hg}$ in group $1 \mathrm{and}$ to 17.0 (13.0; 20.0) $\mathrm{mm} \mathrm{Hg}$ in group 2. At the final visit, IOP levels were within target ranges in both groups, i.e., $13.0(11.0 ; 18.5) \mathrm{mm} \mathrm{Hg} \mathrm{and} \mathrm{13.5} \mathrm{(9.7;} 17.2) \mathrm{mm} \mathrm{Hg}$, respectively. Meanwhile, changes in IOP measured by various methods were significantly different. IOP reduced by $5.4 \%$ (-7.1\%; $17.6 \%)$ in group 1 and by 20.7\% (4.4\%; 30.7\%) in group 2 ( $p<0.05)$ as measured by elastotonometry and by 8.3\% (-11.8\%; 28.6\%) in group 1 and by $33.3 \%(13.9 \% ; 50.7 \%)$ in group 2 as measured by iCare tonometer.
\end{abstract}


Conclusions: brimonidine $0.2 \%$ provides additional IOP reduction to enhance maximum tolerated medical therapy for advanced POAG. An estimated effect of brimonidine is a $8 \%$ reduction of IOP from the baseline. If target IOP is not achieved, a patient should be scheduled for surgery. IOP should be measured using the iCare tonometer since this device is more sensitive to minor IOP fluctuations.

Keywords: brimonidine $0.2 \%$, maximum tolerated medical therapy for glaucoma, tonometry, elastotonometry, glaucoma, trabeculectomy, additive effect, target IOP.

For citation: Dorofeev D.A., Balukhtina V.P., Es'kova M.V. et al. Additive effect of brimonidine in maximum tolerated medical therapy for primary open-angle glaucoma. Russian Journal of Clinical Ophthalmology. 2021;21(3):129-134(in Russ.). DOI: 10.32364/2311-7729-2021-21-3-129-134.

\section{ВВЕДЕНИЕ}

За последние 10 лет отмечается рост выявляемости глаукомы на начальных стадиях. Так, в 2011 г., по данным Российского глаукомного общества, более чем в $60 \%$ случаев глаукома выявлялась на развитой и далеко зашедшей стадиях [1, 2], а в 2021 г., по данным тех же авторов, количество указанных стадий уменьшилось до 50\% [3]. Считается, что в среднем на фоне проводимого лечения переход от одной стадии

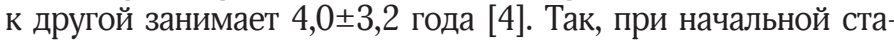
дии глаукомы переход на следующую стадию происходит через 3,1-4,5 года, при развитой стадии - через 2,9-4,3 года, а при далеко зашедшей - через 1,8-3,5 года [5-10].

Выявление глаукомы на развитой и далеко зашедшей стадиях и быстрое прогрессирование глаукомного процесса часто требуют раннего назначения комбинированной терапии, уже на старте наблюдения [6], однако стабилизация глаукомного процесса не всегда зависит от выбранной тактики лечения, а зачастую обусловлена самой стадией заболевания, что, в свою очередь, предопределяет выбор агрессивной терапии и ранний переход к максимальной медикаментозной терапии.

Максимальная медикаментозная терапия (ММТ) это терапия, подразумевающая использование трех и более классов местных гипотензивных агентов, что позволяет достичь максимального снижения ВГД при минимизации побочных эффектов и проблем, с соблюдением рекомендаций врача $[11,12]$. Несмотря на то, что при отсутствии компенсации ВГД на 2-3-х препаратах рекомендуется рассмотреть вопрос о лазерном или хирургическом лечении, усиление ММТ 4-м препаратом является актуальным для ряда пациентов, которым проведение хирургического лечения невозможно по различным причинам (местные или общие противопоказания, эпидемиологическая обстановка в регионе и т. д.).

В качестве дополнительного гипотензивного агента зачастую используется бримонидин 0,2\%. Это селективный $\alpha 2$-адреномиметик, который снижает ВГД за счет уменьшения продукции водянистой влаги и увеличения увеосклерального оттока [13]. По гипотензивной эффективности бримонидин $0,2 \%$ сопоставим с тимололом 0,5\% [14]. К тому же в исследованиях на лабораторных животных было выявлено, что бримонидин обладает также нейропротективным действием [15].

Так как работ по исследованию эффективности добавления бримонидина в качестве 4-го препарата к ММТ практически нет [16], целью нашего исследования стала оценка аддитивного эффекта снижения ВГД бримонидином 0,2\% для достижения давления цели при усилении максимального режима гипотензивной терапии первичной открытоугольной глаукомы (ПОУГ).

\section{МАТЕРИАЛ И МЕТОДЫ}

В период с августа 2018 г. по май 2021 г. были обследованы 63 пациента (63 глаза), средний возраст которых составлял 73,0 $(64,5 ; 77,5)$ года, с развитой стадией ПОУГ. Исследование было проспективным открытым сравнительным в двух параллельных группах.

Критерии включения: развитая стадия ПОУГ; выявленная субкомпенсация ВГД на фоне гипотензивной терапии аналогами простагландинов вместе с фиксированной комбинацией ингибитора карбоангидразы и бета-блокатора; возраст на момент включения в исследование - от 45 до 89 лет; регион проживания - г. Челябинск; клиническая рефракция в диапазоне $\pm 3,0$ дптр и астигматизм $\pm 1,5$ дптр; толщина роговицы в центральной зоне не учитывалась.

Критерии исключения: любая иная форма глаукомы; помутнение оптических сред, препятствующее выполнению периметрических исследований с помощью стандартной автоматической периметрии; другие заболевания сетчатки (возрастная макулодистрофия, состояния после окклюзий сосудов сетчатки, диабетическая ретинопатия и ее осложнения; наличие в анамнезе осложнений оперативного лечения офтальмопатологии; травмы и заболевания органа зрения и его придаточного аппарата; другие общие заболевания, требующие гормональной терапии.

Все пациенты проходили офтальмологическое обследование, включающее визорефрактометрию, биомикроофтальмоскопию, гониоскопию, тонометрию с помощью портативного офтальмологического тонометра iCare (Icare Finland Оу, Финляндия), эластотонометрию, статическую автоматическую компьютерную периметрию (Octopus 600, Haag-Streit International, Koeniz, Switzerland), оптическую когерентную томографию и пахиметрию на приборе Revo NX (Optopol Technology SA, Zawiercie, Poland).

На момент включения в исследование (визит 1) к имеющемуся режиму ММТ у всех пациентов добавлялся препарат группы $\alpha 2$-адреномиметиков - бримонидин $0,2 \%$. Через 1 мес. пациенты приглашались повторно (визит 2), где после офтальмологического обследования по вышеуказанному плану оценивалась компенсация ВГД. В зависимости от степени снижения ВГД исследуемых делили на 2 группы: в 1-ю группу были включены пациенты, у которых удалось достичь целевого уровня ВГД; во 2-ю группу вошли пациенты с сохраняющейся субкомпенсацией, которым затем была выполнена синустрабекулэктомия (СТЭ). В сроки не ранее чем через 2 мес. от визита 2 пациентам назначалось заключительное обследование (визит 3).

Статистическую обработку полученных данных проводили с использованием программного обеспечения R Core Team (2016). R: A language and environment for statistical computing (Vienna, Austria). Приводимые параметры (нормальность распределения проверяли с помощью теста Шапиро - Уилка, гомогенность дисперсии с помощью теста Бартлетта) представлены в формате Ме $\left(\mathrm{Q}_{25 \%} ; \mathrm{Q}_{75 \%}\right)$, где $\mathrm{Me}$ - медиана, $\mathrm{Q}_{25 \%}$ и $\mathrm{Q}_{75 \%}$ - квартили. При сравнении нескольких выборок использовали критерий Уилкоксона. Критический уровень значимости при проверке статистических гипотез принимали равным меньше 0,05. 
Таблица 1. Клинико-демографическая характеристика пациентов по группам

Table 1. Clinical and demographic characteristics of patients

\begin{tabular}{|c|c|c|}
\hline Критерий / Criteria & Группа 1 / Group 1 (n=39) & Группа 2 / Group 2 (n=24) \\
\hline Возраст, лет / Age, years Me (Q25\%; Q75\%) & $71,0(65,0 ; 75,5)$ & $75,0(64,5 ; 80,0)$ \\
\hline Анамнез, лет / Anamnesis, years Me (Q25\%; Q75\%) & $5,7(2,95 ; 11,5)$ & $3,5(2,6 ; 8,8)$ \\
\hline Время контроля, лет / Follow-up, yearsMe (Q25\%; Q75\%) & $0,1(0,1 ; 0,35)$ & $0,1(0,1 ; 0,22)$ \\
\hline Пол (ж/м) / Gender (F/M) & $26 / 13$ & $14 / 10$ \\
\hline Глаз (OD/OS) / Eye (OD/OS) & $20 / 19$ & $8 / 16$ \\
\hline иол (да/нет) / IOL (yes/no) & $18 / 21$ & $14 / 10$ \\
\hline СТЭ (да/нет)* / Trabeculectomy (yes/no) ${ }^{*}$ & 16 (в анамнезе/prior)/23 & $24 / 0$ \\
\hline Наследственность (да/нет) / Heredity (yes/no) & $8 / 31$ & $6 / 18$ \\
\hline СД (да/нет) / Diabetes (yes/no) & $4 / 35$ & $2 / 22$ \\
\hline ПЭС (да/нет) / XFS (yes/no) & $25 / 14$ & $11 / 13$ \\
\hline ЦТТ, мкм / ССТ, $\mu \mathrm{m}$ & $520(502 ; 542)$ & $516(498 ; 541)$ \\
\hline MD, dB / Me (Q25\%; Q75\%) & $-12,4(-20,9 ;-6,0)$ & $-9,45(-13,5 ;-6,0)$ \\
\hline PSD, dB / Me (Q25\%; Q75\%) & $7,2(5,85 ; 8,2)$ & $6,15(3,3 ; 7,7)$ \\
\hline CHBC, MKм / RNFL, $\mu \mathrm{m}$ Me (Q25\%; Q75\%) & $68,0(62,0 ; 75,0)$ & $67,5(59,0 ; 79,0)$ \\
\hline
\end{tabular}

Примечание. ИОЛ - интраокулярная линза, СД - сахарный диабет, ПЭС - псевдоэксфолиативный синдром, ЦТР - центральная толщина роговицы, $M D$ - среднее отклонение светочувствительности сетчатки, PSD - стандартное отклонение средней светочувствительности сетчатки, СНВC - слой нервных волокон сетчатки, * - межгрупповые различия достоверны (p<0,05), по остальным указанным признакам уровень статистической значимости достигнут не был ( $p>0,05)$.

Note. IOL, intraocular lens; XFS, exfoliation syndrome; CCT, central corneal thickness; MD, mean deviation; PSD, pattern standard deviation; RNFL, retinal nerve fiber layer. ${ }^{*}$, intergroup differences are significant $(p<0.05)$.

Таблица 2. Сравнительная оценка уровня ВГД по данным тонометра iCare и эластотонометрии

Table 2. IOP levels measured using iCare tonometer or by elastotonometry

\begin{tabular}{|c|c|c|c|c|}
\hline \multirow{2}{*}{\multicolumn{2}{|c|}{$\begin{array}{l}\text { Номер визита } \\
\text { No. of visit } \\
\text { Method of IOP measurement }\end{array}$}} & \multicolumn{2}{|c|}{$\begin{array}{c}\text { Уровень ВГД, мм рт. ст. / p-value при внутригрупповом сравнении } \\
\text { ІОР, mm Hg / p (intragroup comparison) }\end{array}$} & \multirow{2}{*}{$\begin{array}{c}\text { p-value } \\
\text { при межгрупповом } \\
\text { сравнении } \\
\text { p (intergroup } \\
\text { comparison) }\end{array}$} \\
\hline & & $\begin{array}{c}\text { Группа } 1 \\
\text { Group } 1 \text { (n=39) }\end{array}$ & $\begin{array}{c}\text { Группа } 2 \\
\text { Group } 2(n=24)\end{array}$ & \\
\hline \multirow{4}{*}{ V1 } & $\mathrm{P}_{0}$ & $17,0(12,0 ; 19,0)$ & $19,5(15,8 ; 23,5)$ & $\mathrm{p}<0,05$ \\
\hline & $P_{5}$ & $18,5(16,2 ; 21,2)$ & $19,0(17,2 ; 21,0)$ & $p>0,05$ \\
\hline & $P_{10}$ & $23,5(21,2 ; 26,5)$ & $24,2(22,5 ; 27,9)$ & $\mathrm{p}>0,05$ \\
\hline & $P_{15}$ & $28,5(26,2 ; 30,5)$ & $28,5(26,4 ; 32,2)$ & $p>0,05$ \\
\hline \multirow{4}{*}{ V2 } & $P_{0}$ & $14,0(9,5 ; 17,0) / p_{v 2-v 1}=0,007$ & $17,0(13,0 ; 20,0) / p_{v 2-v 1}=0,05$ & $\mathrm{p}<0,05$ \\
\hline & $\mathrm{P}_{5}$ & $17,5(16,0 ; 19,5) / \mathbf{p}_{\mathrm{v}^{2}-\mathrm{v}_{1}}=\mathbf{0 , 0 3}$ & $18,2(16,0 ; 21,0) / p_{v_{2}-v_{1}}=0,3$ & $p>0,05$ \\
\hline & $P_{10}$ & $21,5(20,0 ; 24,2) / \mathrm{p}_{\mathrm{v} 2-\mathrm{v} 1}=0,0003$ & $23,8(21,4 ; 25,1) / p_{v_{2}-v_{1}}=0,5$ & $p<0,05$ \\
\hline & $P_{15}$ & $27,0(25,0 ; 28,5) / \mathbf{p}_{\mathrm{v} 2-\mathrm{v} 1}=\mathbf{0 , 0 0 9}$ & $27,2(26,0 ; 30,1) / p_{V 2-V 1}=0,3$ & $p>0,05$ \\
\hline \multirow{4}{*}{ V3 } & $P_{0}$ & $13,0(11,0 ; 18,5) / p_{\mathrm{v}^{-}-\sqrt{ } 2}=0,2$ & $13,5(9,7 ; 17,2) / \mathbf{p}_{\mathrm{v} 3-\mathrm{v} 2}=\mathbf{0 , 0 1}$ & $p>0,05$ \\
\hline & $P_{5}$ & $17,0(15,0 ; 19,2) / p_{v_{3}-v 2}=0,9$ & $16,0(14,2 ; 19,7) / p_{\mathrm{V}^{-}-\mathrm{V} 2}=0,1$ & $p>0,05$ \\
\hline & $P_{10}$ & $21,5(20,0 ; 24,5) / p_{v_{3}-v_{2}}=0,2$ & $20,5(18,5 ; 23,6) / \mathbf{p}_{\mathrm{v} 3-\mathrm{v} 2}=\mathbf{0 , 0 2}$ & $p>0,05$ \\
\hline & $P_{15}$ & $26,5(24,7 ; 30,0) / p_{v_{3}-v_{2}}=0,5$ & $26,5(24,7 ; 29,1) / p_{\mathrm{v}^{3}-\mathrm{v} 2}=0,2$ & $p>0,05$ \\
\hline
\end{tabular}

Примечание. Ро - уровень офртальмотонуса, измеренный тонометром іCare, P5/10/15 - уровень офртальмотонуса по данным эластотонометрии (5/10/15 г). Note. PO, IOP measured using iCare tonometer; P5/10/15, IOP measured by elastotonometry (5/10/15 g).

\section{РЕЗУЛЬТАТЫ И ОБСУЖДЕНИЕ}

Клинико-демографическая характеристика пациентов, включенных в исследование, представлена в таблице 1.

Общая продолжительность наблюдения за пациентами составила $0,2(0,1 ; 0,65)$ года. Напряженная эпидемиологическая обстановка в регионе не позволила провести заключительный визит в фиксированные сроки, в связи с чем длительность наблюдения пациентов варьировала: в 1 -й группе она составила $0,1(0,1 ; 0,5)$ года, во 2-й $0,4(0,17 ; 0,7)$ года $(\mathrm{p}>0,05)$.

После разделения пациентов на группы стало возможным оценить ретроспективно средний по обеим группам 


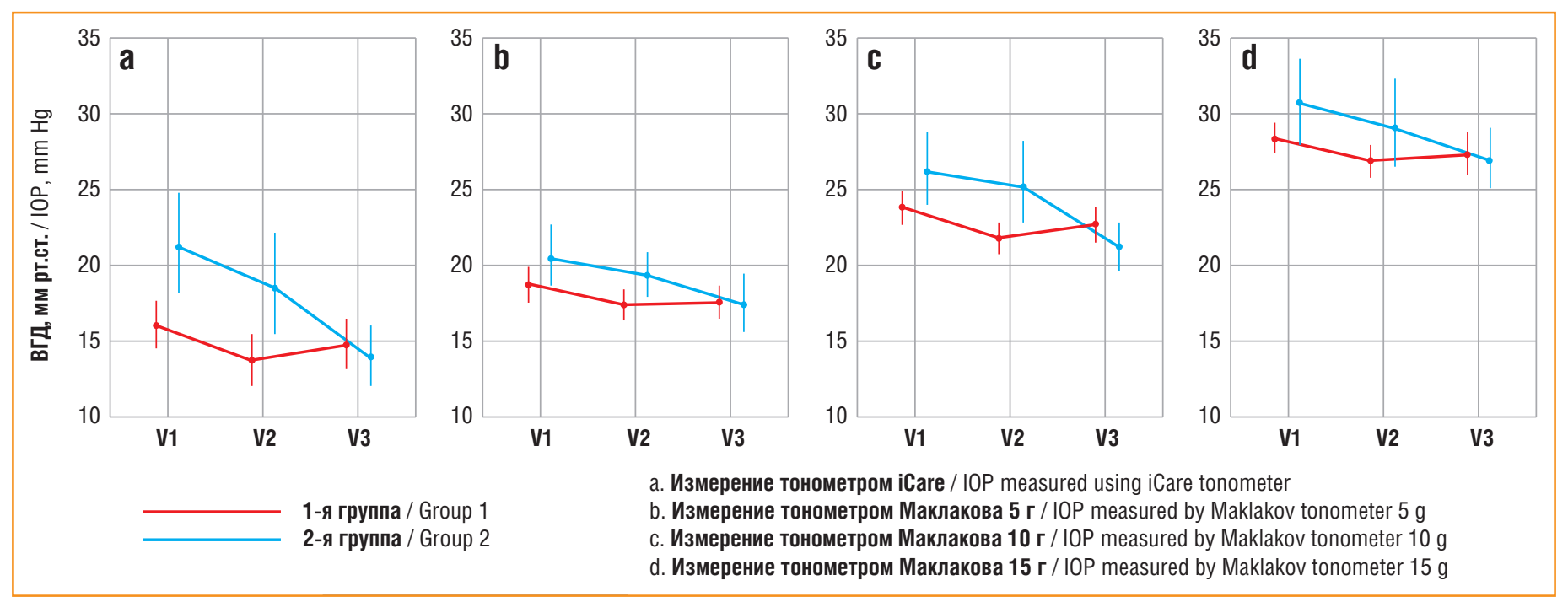

Рис. 1. Динамика уровня ВГД по данным тонометрии и эластотонометрии

Fig. 1. Changes in IOP levels measured by tonometry and elastonometry

уровень ВГД на визите 1: у пациентов группы оперативного лечения уровень офтальмотонуса оказался значимо выше, чем в группе консервативного лечения, но только по данным тонометра iCare (табл. 2).

Через 1 мес. после усиления гипотензивного режима бримонидином 0,2\% в обеих группах наблюдалось статистически значимое снижение офтальмотонуса: по данным всех тонометров в группе консервативного лечения и только по данным тонометра iCare в группе оперативного лечения (см. табл. 2).

На заключительном обследовании у пациентов 1-й группы отмечалось сохранение достигнутого уровня истинного ВГД в пределах целевых значений, значимых различий с показателями ВГД, полученными на визите 2, выявлено не было. У пациентов же 2-й группы отмечалось статистически значимое снижение ВГД в сравнении с показателями на визите 2 по данным тонометров iCare и Маклакова 10 г (см. табл. 2).

Сравнительный анализ показателей офтальмотонуса в течение всего периода наблюдения наглядно отражает схожую динамику по данным использованных методов измерения ВГД (рис. 1). В обеих группах был достигнут целевой уровень ВГД с сопоставимыми показателями. Однако были выявлены значимые межгрупповые различия в итоговом проценте снижения офтальмотонуса: в 1-й группе снижение ВГД по усредненным для всех тонометров данным составило 5,4\% (-7,1\%; 17,6\%), а во 2-й - 20,7\% (4,4\%; 30,7\%) $(\mathrm{p}<0,05)$. Различия по данным только тонометра iCare были наиболее выраженными и составили 8,3\% (-11,8\%; 28,6\%) для группы консервативного лечения и $33,3 \%$ (13,9\%; $50,7 \%)$ для группы оперативного лечения. В группе 1 через 1 мес. применения бримонидина среднее $\mathrm{P}_{0}$ снизилось с $16,1 \pm 5,0$ до $13,8 \pm 5,7$ мм рт. ст., т. е. на $14,3 \%$, что является хорошим результатом для 4-го гипотензивного препарата.

На сегодняшний день проведен целый ряд исследований, посвященных оценке гипотензивной эффективности бримонидина $0,2 \%$. Так, эффективность монотерапии бримонидином 0,2\% для снижения ВГД, по данным метаанализа 24-часового контроля уровня офтальмотонуса, проведенного Stewart et al. еще в 2007 г., составляет 14\% [17]. В свою очередь, по данным Zimmerman et al., которые упоминаются в публикации, посвященной MМТ, гипотензивная эффективность латанопроста при его добавлении для усиления ММТ в качестве четвертого гипотензивного агента не превышает 1-2 мм рт. ст. [11]. При этом в исследовании Schwartzenberg и Buys при назначении бримонидина 0,2\% пациентам с максимальным режимом гипотензивной терапии офтальмотонус снижался в среднем на $20 \%$, около $50 \%$ пациентов продолжали терапию через 9 мес. при хорошей переносимости препарата [16]. В нашем же исследовании у пациентов с субкомпенсированным офтальмотонусом на ММТ дополнительное назначение бримонидина $0,2 \%$ дало снижение уровня истинного ВГД на 8,3\%. Вероятным объяснением такой разной гипотензивной эффективности максимального медикаментозного режима гипотензивной терапии в нашем исследовании и по данным Schwartzenberg и Buys является характер отбора пациентов.

Разница между показателями офтальмотонуса, полученными разными методами тонометрии, обусловлена особенностями взаимодействия тонометров с биомеханическими свойствами фиброзной оболочки глаза. Это объясняет полученную в процессе измерения разницу в проценте снижения офтальмотонуса по данным тонометра iCare и эластотонометрии [16]. В нашем исследовании средние значения ВГД, измеренного тонометром Маклакова (10 г), были не менее 20,5 (18,5; 23,6) мм рт. ст., а при измерении ВГД тонометром iСare среднее значение составило 13,0 (11,0; 18,5). Меньшая разница в динамике офтальмотонуса в послеоперационном периоде по данным эластотонометрии, вероятнее всего, обусловлена ограничением метода тонометрии по Маклакову, которое выражается в том, что идентифицировать офтальмотонус меньше 20 мм рт. ст. таким методом у большого числа пациентов невозможно (это можно увидеть на примере многоцентровых исследований). Так, по данным многочисленных исследований группы «Научный авангард», в клинической практике крайне редко фиксируются значения среднего уровня офтальмотонуса по данным тонометрии по Маклакову (10 г) менее 20 мм рт. ст. [7-10, 18-34].

\section{ЗАКЛЮЧЕНИЕ}

Проведенное исследование демонстрирует аддитивный эффект бримонидина 0,2\% при усилении максимального режима гипотензивной терапии развитой стадии ПОУГ. 
При достижении целевого уровня ВГД в раннем периоде аддитивной терапии отмечается стойкое сохранение достигнутого результата. Если аддитивный эффект бримонидина $0,2 \%$ недостаточен, проведение дополнительно антиглаукомной операции позволяет снизить офтальмотонус до целевого уровня. При этом получаемые показатели ВГД сопоставимы в обоих случаях $(\mathrm{p}>0,05)$.

Таким образом, при добавлении к ММТ 4-го гипотензивного препарата - бримонидина $0,2 \%$ необходимо учитывать предполагаемый гипотензивный эффект - дополнительное снижение истинного ВГД в среднем на 8\%, и, если это снижение не позволяет достичь целевого уровня, планировать оперативное вмешательство. При терапии субкомпенсированного ВГД у пациентов на ММТ предпочтительнее проводить измерение тонометром iCare как более чувствительным к небольшим перепадам офтальмотонуса.

\section{Лumepamypa}

1. Егоров Е.А., Куроедов А.В. Отдельные клинико-эпидемиологические характеристики глаукомы в странах СНГ и Грузии. Результаты многоцентрового открытого ретроспективного исследования (часть 1). РМЖ. Клиническая офтальмоло гия. 2011:12(3):97-100.

2. Егоров Е.А., Куроедов А.В. Отдельные клинико-эпидемиологические характеристики глаукомы в странах СНГ и Грузии. Результаты многоцентрового открытого ретроспективного исследования (часть 2). РМЖ. Клиническая офтальмология. 2011:12(1):13-22.

3. Куроедов А.В., Мовсисян А.Б., Егоров Е.А. и др. Профиль пациентов с первичной открытоугольной глаукомой в Российской Федерации (предварительные результаты многоцентрового популяционного исследования). Часть 1. Национальный журнал глаукома. 2021;20(1):3-15. DOI: 10.25700/NJG.2021.01.01.

4. Абышева Л.Д., Авдеев Р.В., Александров А.С. и др. Многоцентровое исследование по изучению показателей офтальмотонуса у пациентов с продвинутым стадиями первичной открытоугольной глаукомы на фоне проводимого лечения. Офтальмологические ведомости. 2015;8(1):43-60.

5. Нероев В.В., Киселева О.А., Бессмертный А.М. Основные результаты мультицентрового исследования эпидемиологических особенностей первичной открытоугольной глаукомы в Российской Федерации. Российский офтальмологический журнал. 2013;6(3):4-7.

6. Куроедов А.В., Брежнев А.Ю., Ловпаче Д.Н. и др. Целесообразность применения дифференцированных («ступенчатых») стартовых подходов к лечению больных с разными стадиями глаукомы. Национальный журнал глаукома. 2018;17(4):25 54. DOI: $10.25700 / \mathrm{NJG} .2018 .04 .03$.

7. Городничий В.В., Дорофеев Д.А., Завадский П.Ч. и др. Факторы риска, патогенные факторы развития и прогрессирования глаукомы по результатам многоцентрового исследования Российского глаукомного общества. Медико-биологические проблемы жизнедеятельности. 2012;8(2):57-69.

8. Авдеев Р.В., Александров А.С., Басинский А.С. и др. Клинико-эпидемиологическое исследование факторов риска развития и прогрессирования глаукомы. Российский офтальмологический журнал. 2013;6(3):4-11.

9. Абышева Л.Д., Авдеев Р.В., Александров А.С. и др. Оптимальные характеристики верхней границы офтальмотонуса у пациентов с развитой стадией первично открытоугольной глаукомы с точки зрения доказательной медицины. РМЖ. Клиническая офтальмология. 2015;15(3):111-123.

10. Куроедов А.В., Абышева Л.Д., Александров А.С. и др. Тактика ведения пациентов с первичной открытоугольной глаукомой на практике: варианты медикаментозного, лазерного и хирургического лечения. Медико-биологические проблемы жизнедеятельности. 2016;15(1):170-185.

11. Zimmerman T.J. Maximal Medical Therapy for Glaucoma. Arch Ophthalmol. 1997;115(12):1579. DOI: 10.1001/archopht.1997.01100160749014.

12. Fechtner R.D., Singh K. Maximal Glaucoma Therapy. J Glaucoma. 2001;10(1):73-75. DOI: 10.1097/00061198-200110001-00026

13. Toris C.B., Gleason M.L., Camras C.B., Yablonski M.E. Effects of Brimonidine on Aqueous Humor Dynamics in Human Eyes. Arch Ophthalmol. 1995;113(12):1514-1517 DOI: 10.1001/archopht.1995.01100120044006.

14. Schuman J.S. Clinical experience with brimonidine $0.2 \%$ and timolol $0.5 \%$ in glaucoma and ocular hypertension. Surv Ophthalmol. 1996;41(1):27-37. DOI: 10.1016/ s0039-6257 (96) 82029-7.

15. Cantor L.B., Burke J. Brimonidine. Expert Opin Investig Drugs. 1997;6(8):10631083. DOI: $10.1517 / 13543784.6 .8 .1063$

16. Schwartzenberg G.W., Buys Y.M. Efficacy of brimonidine $0.2 \%$ as adjunctive therapy for patients with glaucoma inadequately controlled with otherwise maximal medical therapy. Ophthalmology. 1999;106(8):1616-1620. DOI: 10.1016/S0161-6420 (99) 90461-X

17. Stewart W.C., Konstas A.G.P., Nelson L.A., Kruft B. Meta-analysis of 24hour intraocular pressure studies evaluating the efficacy of glaucoma medicines. Ophthalmology. 2008;115(7):1117-1122. DOI: 10.1016/j.ophtha.2007.10.004.
18. Куроедов А.В., Абышева Л.Д., Авдеев Р.В. и др. Уровни внутриглазного давления при различном местном гипотензивном лечении при первичной открытоугольной глаукоме (многоцентровое исследование). Офтальмология. Восточная Европа. 2016;28(1):27-42.

19. Абышева Л.Д., Авдеев Р.В., Александров А.С. и др. Многоцентровое исследование по изучению показателей офтальмотонуса у пациентов с продвинутыми стадиями первичной открытоугольной глаукомы на фоне проводимого лечения. Новости глаукомы. 2016;37(1):72-81.

20. Куроедов А.В., Абышева ЛД, Авдеев Р.В. и др. Резудьтаты изучения соотношения эффективности и стоимости затрат при применении местной гипотензивной терапии у пациентов с развитой и далеко зашедшей стадиями первичной открытоугольной глаукомы (многоцентровое исследование). Новости глаукомы. 2016:37(1):123-132.

21. Авдеев Р.В., Басинский А.С., Блюм Е.А. и др. Клинико-математическая модель первичной открытоугольной глаукомы манифестирование и исходы. Новости глаукомы 2015:33(1):55-63.

22. Корнеева А.В., Куроедов А.В., Завадский П.Ч. и др. Приверженность гипотензивной терапии при глаукоме: мнение пациентов о ключевых факторах низкой степени комплаенса. Результаты многоцентрового интерактивного научно-аналитического исследования. Национальный журнал глаукома. 2020;19(3):12-21. DOI: $10.25700 / \mathrm{NJG} .2020 .03 .02$

23. Авдеев Р.В., Бакунина Н.А., Басинский А.С. и др. Менеджмент прогрессирования глаукомы. Национальный журнал глаукома. 2019;18(1):45-58. DOI: 10.25700/ NJG.2019.01.07

24. Абышева Л.Д., Александров А.С., Арапиев М.У. и др. Оптимизация лечебно-диагностического процесса у пациентов с первичной открытоугольной глаукомой. Национальный журнал глаукома. 2016;15(2):19-35.

25. Гусаревич А.А., Завадский П.Ч., Куроедов А.В. и др. Актуальность выбора монотерапии аналогами простагландинов/простамидов на старте лечения впервые выявленной глаукомы (результаты многоцентрового исследования). Национальный журнал глаукома. 2020;19(3):43-57. DOI: 10.25700/NJG.2020.03.05,

26. Авдеев Р.В., Александров А.С., Бакунина Н.А. и др. Сопоставление режимов лечения больных первичной открытоугольной глаукомой с характеристиками прогрессирования заболевания. Часть 2. Эффективность инициальных режимов гипотензивного лечения. Национальный журнал глаукома. 2018;17(2):65-83. DOI: $10.25700 /$ NJG.2018.02.07

27. Авдеев Р.В., Александров А.С., Бакунина Н.А. и др. Сопоставление режимов лечения больных первичной открытоугольной глаукомой с характеристиками прогрессирования заболевания. Часть 1 . Состояние показателей офтальмотонуса. Национальный журнал глаукома. 2018;17(1):14-29. DOI: 10.25700 NJG.2018.01.02.

28. Авдеев Р.В., Александров А.С., Басинский А.С. и др. Оценка клинико-инструментальных данных исследования органа зрения у больных первичной открытоугольной глаукомой и макулодистрофией. Медицинский вестник Башкортостана. 2014;9(2):24-28.

29. Авдеев Р.В., Александров А.С., Басинский А.С. и др. Клиническое многоцентровое исследование эффективности синусотрабекулэктомии. Глаукома. Журнал НИИ ГБ РАМН. 2013:(2):53-60.

30. Абышева Л.Д., Авдеев Р.В., Александров А.С. и др. Результаты многоцентрового исследования по изучению стоимости и «стоимости-эффективности» лечения пациентов с глаукомой. Х Съезд офтальмологов России. 2015:34.

31. Авдеев Р.В., Александров А.С., Бакунина Н.А. и др. Моделирование продолжительности сроков заболевания и возраста пациентов с разными стадиями первичной открытоугольной глаукомы. Точка зрения Восток - 3апад. 2014;(1):94-95.

32. Куроедов А.В., Абышева Л.Д., Авдеев Р.В. и др. Результаты изучения соотношения эффективности и стоимости затрат при применении местной гипотензивной терапии у пациентов с развитой и далеко зашедшей стадиями первичной открытоугольной глаукомы (многоцентровое исследование). Российский офтальмологический журнал. 2015;8(3):10-22.

33. Куроедов А.В., Абышева Л.Д., Авдеев Р.В. и др. Показатели офтальмотонуса на фоне различных схем местной гипотензивной терапии у больных с первичной открытоугольной глаукомой (многоцентровое исследование). Проблемы здоровья и экологии. 2015;44(2):23-32.

34. Куроедов А.В., Абышева Л.Д., Авдеев Р.В. и др. Эффективность и затраты на местную гипотензивную терапию у пациентов с первичной открытоугольной глаукомой в странах СНГ. Проблемы здоровья и экологии. 2015;43(1):28-38.

\section{References}

1. Egorov E.A., Kuroyedov A.V. Clinical and epidemiological characteristics of glaucoma in CIS and Georgia. Results of multicenter opened retrospective trial (part 1). Russian Journal of Clinical Ophthalmology. 2011;12(3):97-100 (in Russ.).

2. Egorov E.A., Kuroyedov A.V. Clinical and epidemiological characteristics of glaucoma in CIS and Georgia. Results of multicenter opened retrospective trial (part 2). Russian Journal of Clinical Ophthalmology. 2011;12(1):13-22 (in Russ.).

3. Kuroyedov A.V., Movsisyan A.B., Egorov E.A. et al. The profile of patients with primary open-angle glaucoma in the Russian Federation (preliminary results of a multicenter population-based study). Part 1. National Journal of Glaucoma. 2021;20(1):3-15 (in Russ.). DOI: 10.25700/NJG.2021.01.01

4. Abysheva L.D., Avdeev R.V., Aleksandrov A.S. et al. Multicenter study of intraocular pressure level in patients with moderate and advanced primary open-angle glaucoma on treatment. Ophthalmology Journal. 2015;8(1):43-60 (in Russ.) 
5. Neroev V.V., Kiseleva O.A., Bessmertny A.M. The Main Results of a Multicenter Study of Epidemiological Features of Primary Open-Angle Glaucoma in the Russian Federation. Russian Ophthalmological Journal. 2013;6(3):4-7 (in Russ.)

6. Kuroyedov A.V., Brezhnev A. Yu., Lovpache J.N. et al. The feasibility of adopting "stepwise» initial approaches in treatment of patients with different stages of glaucoma. National Journal of Glaucoma. 2018;17(4):25-54 (in Russ.). DOI: 10.25700/ NJG.2018.04.03.

7. Gorodnichii V.V., Dorofeev D.A., Zavadskii P.C. et al. Risk factors, pathogenic factor in progression of glaucoma by results of multicenter study of Russian glaucoma society. Medical and biological problems of life activity. 2012;8(2):57-69 (in Russ.).

8. Avdeev R.V., Alexandrov A.S., Basinsky A.S. et al. A Clinical and Epidemiological Study of Risk Factors of Glaucoma Development and Progression. Russian Ophthalmological Journal. 2013;6(3):4-11 (in Russ.)

9. Abysheva L.D., Avdeev R.V., Aleksandrov A.S. et al. Safety characteristics of th established optimal values of the intraocular pressure upper limit in patients with advanced primary open-angle glaucoma in terms of evidence-based medicine. Russian Journal of Clinical Ophthalmology. 2015;15(3):111-123 (in Russ.).

10. Kuroyedov A.V., Abysheva L.D., Aleksandrov A.S. et al. Management of primary open-angle glaucoma in practice: variants of medical, laser and surgical treatment. Medical and biological problems of life activity. 2016;15(1):170-185 (in Russ.).

11. Zimmerman T.J. Maximal Medical Therapy for Glaucoma. Arch Ophthalmol. 1997;115(12):1579. DOI: 10.1001/archopht.1997.01100160749014.

12. Fechtner R.D., Singh K. Maximal Glaucoma Therapy. J Glaucoma. 2001;10(1):73-75 DOI: 10.1097/00061198-200110001-00026.

13. Toris C.B., Gleason M.L., Camras C.B., Yablonski M.E. Effects of Brimonidine on Aqueous Humor Dynamics in Human Eyes. Arch Ophthalmol. 1995;113(12):1514-1517. DOI: 10.1001/archopht.1995.01100120044006.

14. Schuman J.S. Clinical experience with brimonidine $0.2 \%$ and timolol $0.5 \%$ in glaucoma and ocular hypertension. Surv Ophthalmol. 1996;41(1):27-37. DOI: 10.1016/ s0039-6257 (96) 82029-7.

15. Cantor L.B., Burke J. Brimonidine. Expert Opin Investig Drugs. 1997;6(8):10631083. DOI: $10.1517 / 13543784.6 .8 .1063$.

16. Schwartzenberg G.W., Buys Y.M. Efficacy of brimonidine $0.2 \%$ as adjunctive therapy for patients with glaucoma inadequately controlled with otherwise maxima medical therapy. Ophthalmology. 1999;106(8):1616-1620. DOI: 10.1016/S0161-6420 (99) 90461-X.

17. Stewart W.C., Konstas A.G.P., Nelson L.A., Kruft B. Meta-analysis of 24 hour intraocular pressure studies evaluating the efficacy of glaucoma medicines. Ophthalmology. 2008;115(7):1117-1122. DOI: 10.1016/j.ophtha.2007.10.004

18. Kuroyedov A.V., Abysheva L.D., Avdeev R.V. et al. Intraocular pressure level in various local antihypertensive therapy in primary open-angle glaucoma (multicenter study). Ophthalmology. Eastern Europe. 2016;28(1):27-42 (in Russ.).

19. Abysheva L.D., Avdeev R.V., Alexandrov A.S. et al. Multicenter study of intraocula pressure level in patients with moderate and advanced primary open-angle glaucoma on treatment. Glaucoma News. 2016;37(1):72-81 (in Russ.)

20. Kuroyedov A.V., Abysheva L.D., Avdeev R.V. et al. Cost-effectiveness medical IOP-lowering treatment study in patients with moderate and advanced primary-open glaucoma (multicenter study). Glaucoma News. 2016;37(1):123-132 (in Russ.).

21. Avdeev R.V., Basinsky A.S., Blyum E.A. et al. Clinical and mathematical model of primary open-angle glaucoma, manifestation and outcomes. Glaucoma News. 2015;33(1):55-63 (in Russ.).

22. Korneeva A.V., Kuroyedov A.V., Zavadsky P. Ch. Adherence to glaucoma hypotensive therapy: patients' opinions on key factors of low compliance. Analytical multi-centra study results. National Journal of Glaucoma. 2020;19(3):12-21 (in Russ.). DOI: 10.25700/ NJG.2020.03.02.

23. Avdeev R.V., Bakunina N.A., Basinsky A.S. et al. Management of glaucoma progression. National Journal of Glaucoma. 2019;18(1):45-58 (in Russ.). DOI 10.25700/NJG 2019.01.07.

24. Abysheva L.D., Aleksandrov A.S., Arapiev M.U. Optimization of diagnosis and treatment options in primary open-angle glaucoma patients. National Journal of Glaucoma. 2016;15(2):19-35 (in Russ.)

25. Gusarevich A.A., Zavadsky P. Ch., Kuroyedov A.V. The correct choice of monotherapy with prostaglandin analogues/prostamides at the start of treatment for newly diagnosed glaucoma (results of a multicenter study). National Journal of Glaucoma. 2020;19(3):4357 (in Russ.). DOI: 10.25700/NJG.2020.03.05.

26. Avdeev R.V., Aleksandrov A.S., Bakunina N.A. et al. Comparison of treatment regimens for patients with primary open-angle glaucoma with the characteristics of disease progression. Part 2. The efficacy of initial antihypertensive treatment regimes. National Journal of Glaucoma. 2018;17(2):65-83 (in Russ.). DOI: 10.25700/ NJG.2018.02.07

27. Avdeev R.V., Aleksandrov A.S., Bakunina N.A. et al. Comparison of treatmen regimens for patients with primary open-angle glaucoma with signs of disease progression. Part 1. IOP levels. National Journal of Glaucoma. 2018;17(1):14-29 (in Russ.). DOI: 10.25700/NJG.2018.01.02.

28. Avdeev R.V., Aleksandrov A.S., Basinsky A.S. et al. Evaluation of clinical and instrumental data of eyes examination in patients with primary open-angle glaucoma and macular degeneration. Bashkortostan medical journal. 2014;9(2):24-28 (in Russ.).

29. Avdeev R.V., Aleksandrov A.S., Basinsky A.S. Clinical multicenter study of trabeculectomy efficacy. Glaucoma. Journal of Research Institute GB RAMS 2013;(2):53-60 (in Russ.)
30. Abysheva L.D., Avdeev R.V., Aleksandrov A.S. et al. Results of a multicenter study examining the cost and «cost-effectiveness» of treatment of patients with glaucoma. X Congress of ophthalmologists of Russia. 2015:34 (in Russ.).

31. Avdeev R.V., Aleksandrov A.S., Bakunina N.A. et al. Modeling the duration of the disease and age with different stages of primary open-angle glaucoma. Point of view. East - West. 2014;(1):94-95 (in Russ.).

32. Kuroyedov A.V., Abysheva L.D., Avdeev R.V. et al. Cost/effectiveness ratio of local hypotensive treatment of patients with moderate and advanced primary open-angle glaucoma (a multicenter study). Russian Ophthalmological Journal. 2015;8(3):10-22 (in Russ.).

33. Kuroyedov A.V., Abysheva L.D., Avdeev R.V. et al. The parameters of intraocular pressure related to different schemes of local hypertensive therapy in patients with primary open-angle glaucoma (multicenter study). Health and ecology issues. 2015;44(2):23-32 (in Russ.).

34. Kuroyedov A.V., Abysheva L.D., Avdeev R.V. The efficiency and cost of loca hypotensive therapy in patients with primary open-angle glaucoma in CIS. Health and ecology issues. 2015;43(1):28-38 (in Russ.)

\section{Сведения об авторах:}

'Дорофеев Дмитрий Александрович - врач-офтальмолог; ORCID iD 0000-0003-3352-8170.

${ }^{2}$ Балухтина Виолетта Петровна - студентка; ORCID iD 0000-0001-7986-9726.

${ }^{1}$ Еськова Марина Владиславовна - оператор OKT; ORCID iD 0000-0001-8321-276X.

${ }^{1}$ Ефимова Ксения Алексеевна - оператор OKT, ORCID iD 0000-0001-6492-7743

${ }^{1}$ Кирилик Елена Викторовна - врач-офтальмолог; ORCID iD 0000-0002-0189-9586.

'Лукьянова Ксения Олеговна - врач-офтальмолог; ORCID iD 0000-0002-6781-3343.

'МАУЗ «ГКБ № 2». 454090, Россия, г. Челябинск, ул. Российская, д. 200

${ }^{2}$ ФГБОУ ВО ЮУГМУ. 454092, Россия, г. Челябинск, ул. Воровского, д. 64.

Контактная информация: Дорофеев Дмитрий Александрович, e-mail:dimmm.83@mail.ru.

Прозрачность финансовой деятельности: никто из авторов не имеет финансовой заинтересованности в представленных материалах или методах. Конфликт интересов отсутствует. Статья поступила 15.06.2021.

About the authors:

${ }^{1}$ Dmitry A. Dorofeev - ophthalmologist; ORCID iD 0000 0003-3352-8170.

${ }^{2}$ Violetta P. Balukhtina - student; ORCID iD 0000-00017986-9726.

${ }^{1}$ Marina V. Es'kova - OCT operator; ORCID iD 0000-00018321-276X.

${ }^{1}$ Kseniya A. Efimova - OCT operator; ORCID iD 0000-00016492-7743.

${ }^{1}$ Elena V. Kirilik - ophthalmologist; ORCID iD 0000-00020189-9586.

${ }^{1}$ Kseniya O. Luk'yanova - ophthalmologist; ORCID iD 0000 0002-6781-3343.

${ }^{1}$ City Clinical Hospital No. 2, Polyclinic № 1; 200, Rossiiskaya St., Chelyabinsk, 454090, Russian Federation.

${ }^{2}$ South Ural State Medical University; 64, Vorovskiy str., Chelyabinsk, 454092, Russian Federation.

Contact information:Dmitry A.Dorofeev, e-mail:dimmm.83@ mail.ru.

Financial Disclosure: no authors have a financial or property interest in any material or method mentioned. There is no conflict of interests. Received 15.06.2021. 